\title{
Stents metálicos autoexpandibles para el manejo endoscópico de las fístulas esofagogástricas
}

\section{Self-expanding metal stents for endoscopic management of esophageal and gastric fistulas}

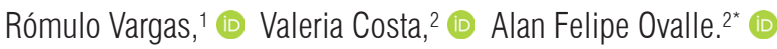

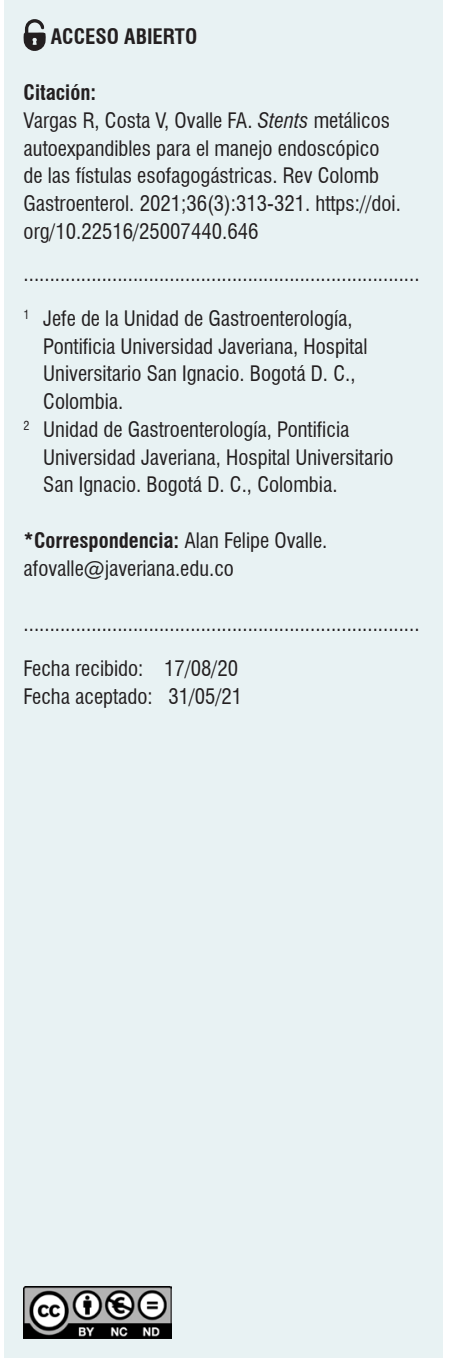

\begin{abstract}
Resumen
Objetivos: mostrar la eficacia y seguridad de los stents metálicos autoexpandibles para el manejo endoscópico de las fístulas esofágicas. Materiales y métodos: se evalúo una serie de casos de manera retrospectiva entre el 2007 y el 2017, en los que se manejaron a 11 pacientes con un stent metálico autoexpandible para el manejo de fístula esofágica, en quienes se realizó el diagnóstico por clínica, endoscopia digestiva alta o estudios radiológicos en la unidad de gastroenterología del Hospital Universitario San Ignacio (HUSI) de Bogotá D. C., Colombia. Resultados: el principal síntoma inicial fue la disnea en $27,3 \%$ de los casos, seguido por tos en un $18,2 \%$. El hallazgo más frecuentemente encontrado durante el seguimiento fue el derrame pleural en el 36,4\%, se realizó el diagnóstico de fístula en el $45,5 \%$ con esofagograma y el tipo de lesión más reportada fue la fuga en la anastomosis esofagoentérica, con un $45,5 \%$, seguida de la esofagopleural, con un $36,4 \%$; y estos pacientes fueron manejados con un stent metálico autoexpandible. En el $100 \%$ hubo éxito técnico y la resolución del defecto se evidenció en el $72,7 \%$ de los casos. La única complicación reportada fue el desplazamiento del stent en el $27,3 \%$, y en un paciente se requirió el cambio del stent en 3 oportunidades. El promedio de estancia hospitalaria fue de 41,5 días. Conclusiones: el manejo endoscópico de las fístulas esofagogástricas con stents metálicos autoexpandibles es efectivo y seguro, con una baja tasa de complicaciones.
\end{abstract}

\section{Palabras clave}

Fístulas esofágicas, stent metálico autoexpandible, anastomosis, complicaciones, cirugía de esófago.

\begin{abstract}
Objective: To demonstrate the efficacy and safety of self-expanding metal stents for endoscopic management of esophageal fistulas. Materials and methods: Retrospective case series between 2007 and 2017. A total of 11 patients were treated with self-expanding metal stents for esophageal fistula management, after being diagnosed based on symptoms, upper endoscopy, and/or radiological studies in the gastroenterology unit of the Hospital Universitario San Ignacio (HUSI) in Bogotá D.C, Colombia. Results: The most common initial symptom was dyspnea in $27.3 \%$ of cases, followed by cough in $18.2 \%$. The most frequent finding during follow-up was pleural effusion in $36.4 \%$ of the cases, of which $45.5 \%$ received a diagnosis of fistula through esophagogram. The most reported lesion was esophagoenteric anastomotic leak with $45.5 \%$, followed by esophagopleural injury with $36.4 \%$; these patients were those who received self-expanding metal stent management. Technical success was achieved in $100 \%$ of the cases, and the defect was resolved in in $72.7 \%$ of them. The only complication reported was stent migration in $27.3 \%$, requiring 3 changes in 1 patient. The average hospital stay was 41.5 days. Conclusions: Endoscopic management of esophagogastric fistulas with self-expanding metal stents is effective and safe, with a low complication rate.
\end{abstract}

\section{Keywords}

Esophageal fistulas; Self-expanding metal stent; Anastomosis; Complications; Esophageal surgery. 


\section{INTRODUCCIÓN}

Las fugas y fístulas gastrointestinales son condiciones graves $y$ potencialmente mortales que pueden verse como una amplia variedad de presentaciones clínicas. Las fugas están relacionadas principalmente con defectos anastomóticos posoperatorios y son responsables de una parte importante de la morbimortalidad quirúrgica. Las fugas y las colecciones posoperatorias pueden conducir al desarrollo de una fístula entre dos estructuras epiteliales. La endoscopia intervencionista con la colocación de un stent juega un papel fundamental como tratamiento de primera línea y de rescate en estas situaciones, ya que es un método eficaz y mínimamente invasivo en el que se requiere un enfoque personalizado y multidisciplinario basado en la presentación clínica, las características del defecto (tamaño, ubicación), experiencia local y la disponibilidad de dispositivos. La fuga se define como una comunicación patológica entre compartimentos intra- y extraluminales, mientas que la fístula se define como una comunicación anormal entre dos superficies epitelializadas ${ }^{(1,2)}$.

La mayoría de las fugas y fístulas gastrointestinales (75\%-85\%) ocurre por una complicación de la cirugía intraabdominal y es causada por una variedad de factores incluidas la ausencia o la incorrecta colocación de drenajes, desnutrición, inadecuada técnica quirúrgica, infección y dehiscencia de anastomosis. Un porcentaje menor ocurre secundariamente a enfermedad inflamatoria intestinal, diverticulitis, neoplasias, trauma y radioterapia ${ }^{(3)}$.

Las fístulas pueden ser difíciles de diagnosticar y demandan frecuentemente reintervenciones quirúrgicas; de este modo aumenta la morbimortalidad y los costos de la hospitalización; por tanto, los principios fundamentales del tratamiento son la identificación del sitio del defecto, el drenaje y la prevención de colecciones adicionales, ya sea mediante la desviación del flujo del contenido luminal o el cierre del defecto $^{(4,5)}$. Aunque el manejo quirúrgico tradicional o el conservador con reposo intestinal, los antibióticos intravenosos y el soporte nutricional son los pilares del tratamiento, no siempre son efectivos ${ }^{(6-8)}$. El manejo endoscópico ha demostrado ser una alternativa eficaz y menos invasiva que la cirugía primaria, y los stents metálicos autoexpandibles total o parcialmente cubiertos son una alternativa mínimamente invasiva en el manejo de las fugas y fístulas gastrointestinales, cuyo objetivo es prevenir la fuga del contenido gastrointestinal por el trayecto fistuloso y, de este modo, permitir la cicatrización del defecto, lo que favorece que el paciente retome la nutrición por vía oral y mejore sus condiciones nutricionales, para que de esta manera se permita el cierre del defecto y, a futuro, mejore la calidad de vida ${ }^{(9,10)}$.
Una de las desventajas de los stents metálicos es que pueden causar hiperplasia de la mucosa adyacente, lo que hace difícil removerlos una vez se ha logrado el cierre de la fístula ${ }^{(10)}$. Los stents no cubiertos o parcialmente cubiertos están más asociados con hiperplasia epitelial y otras complicaciones, especialmente cuando su uso es transitorio, como para el manejo de condiciones benignas. Por tanto, se ha preferido el uso de stents metálicos completamente recubiertos para esta indicación, aunque pueden cursar con una mayor tasa de migración ${ }^{(11)}$.

Blackmon y colaboradores reportaron datos de un estudio prospectivo con seguimiento a 15 meses, que incluyó 25 pacientes, de los cuales 23 pacientes con diagnóstico de fugas anastomóticas, fístulas traqueoesofágicas y perforaciones benignas fueron manejados con stents metálicos cubiertos como primera línea de tratamiento. Se obtuvo curación en 10 pacientes manejados con stent por fuga anastomótica después de bypass gástrico o manga gástrica, un paciente con tres perforaciones iatrogénicas esofágicas tuvo control con la colocación de un stent y en 2 de 4 pacientes con fístula traqueoesofágica se logró sellar la misma con el uso de stents. Como complicación más frecuente se reportó la migración del stent en 10 pacientes ${ }^{(12)}$.

La serie retrospectiva de Tuebergen y colaboradores incluyó 32 pacientes, 24 con fugas posoperatorias, la mayoría luego de cirugía oncológica gastroesofágica y 8 pacientes con perforación no maligna de esófago. Lograron un sellado funcional completo después del despliegue del stent en el $78 \%$ de los casos con el uso de stents metálicos completamente cubiertos, y se destaca que la postura se realizó en promedio entre 3 y 5 días después del diagnóstico ${ }^{(13)}$.

El objetivo primario de este estudio es evaluar las tasas de cierre en el manejo de las fístulas gastrointestinales con stents metálicos autoexpandibles, y como objetivo secundario está determinar las complicaciones tempranas y tardías y la estancia hospitalaria.

\section{MATERIALES Y MÉTODOS}

Se realizó una evaluación retrospectiva de las bases de datos de los procedimientos intervencionistas de la unidad de gastroenterología, desde enero de 2007 hasta diciembre de 2017, y se encontraron 11 pacientes con diagnóstico de fístula esofágica, de los cuales 10 fueron tratados con un stent metálico autoexpandible (SMA) y 1 con SMA más OVESCO (Over The Scope Clip) en la unidad de gastroenterología del Hospital Universitario San Ignacio. Todos los datos fueron registrados en un formato diseñado para tal fin, antes y después de la colocación del stent. El seguimiento se hizo bajo la revisión de las historias clínicas o por contacto telefónico. 
Con respecto al diagnóstico de base, en dos casos se reportó el antecedente de adenocarcinoma y un carcinoma escamocelular del tercio distal del esófago (3 casos, $27 \%)$. En 6 casos (55\%) hubo adenocarcinomas del cardias ( 1 caso) o del cuerpo gástrico (5 casos), un caso de cirugía por obesidad $(9 \%)$ y uno de cáncer de cérvix metastásico perforado (9\%) (Figura 1). Derivado de las patologías de base anteriormente descritas, el manejo quirúrgico más frecuentemente realizado fue la gastrectomía total más esofagoyeyunostomía en Y de Roux (45,5\%), seguido por esofagogastrectomía más anastomosis esofagogástrica $(36,4 \%)$.

Los parámetros clínicos se registraron usando una base de datos de Microsoft Excel, que describen el éxito técnico, éxito clínico, complicaciones relacionadas con el procedimiento tales como el desplazamiento del SMA, mortalidad y la duración de la estancia hospitalaria. Los pacientes tuvieron seguimiento con estudios endoscópicos y radiológicos.

El consentimiento informado para dicho procedimiento se obtuvo de todos los pacientes o sus familiares, en caso de que el paciente no pudiese dar la autorización por la condición clínica del momento. En todos los pacientes la colocación del SMA se realizó bajo sedación suministrada por anestesiología, con estricta monitorización de los signos vitales. La localización del defecto esofágico se marcó para su identificación bajo fluoroscopia en la superficie de la piel con un identificador radiopaco. Luego, se insertó una guía semirrígida en la luz esofágica bajo visión endoscópica y se dejó in situ con retiro del gastroscopio, se introdujo sobre la guía un stent de nitinol total o parcialmente cubierto, el cual se liberó bajo visión fluoroscópica y endoscópica y se verificó que la localización de la fístula quedara en la parte media del SMA (éxito técnico) por medio de una endoscopia digestiva alta.

La extracción del SMA se realizó en promedio a las 4 semanas, en todos los pacientes que tuvieron evidencia de cicatrización del trayecto fistuloso corroborado mediante la realización de una radiografía de vías digestivas altas. El éxito clínico se definió como la ausencia de síntomas (disnea, tos, expectoración y disfagia), con la normalización de los marcadores séricos de inflamación, y evidencia endoscópica o radiológica del control del producido de la fístula.

\section{RESULTADOS}

Se colocaron 14 SMA en 11 pacientes, que se distribuyeron en 6 mujeres $(54,5 \%)$ con una edad promedio de 58 años (desviación estándar [DE]: 16,02), con un mínimo de 36 años y un máximo de 86 años. Con respecto a las comorbilidades encontradas, la mayoría de los pacientes no presentaba ninguna $(63,6 \%)$, dos pacientes tenían hipertensión arterial (HTA) y diabetes mellitus (18,2\%), un paciente con hipertensión y otra con antecedente de cáncer de cérvix.

En cuanto a las características clínicas de los pacientes incluidos en el estudio (Tabla 1), se encontró que el principal síntoma fue la presencia de disnea en 3 casos $(27,3 \%)$, seguido por tos en 2 casos $(18,2 \%)$ y los 6 pacientes restantes presentaron cada uno síntomas diferentes, que se muestran en la Figura 2. El tiempo de evolución de los síntomas en la mayoría de casos fue subagudo (7-15 días; 63,6 \%), seguido por síntomas crónicos (> 15 días; 27,3\%).

El hallazgo más frecuentemente encontrado durante el seguimiento fue el derrame pleural $(36,4 \%)$ y se realizó el
A

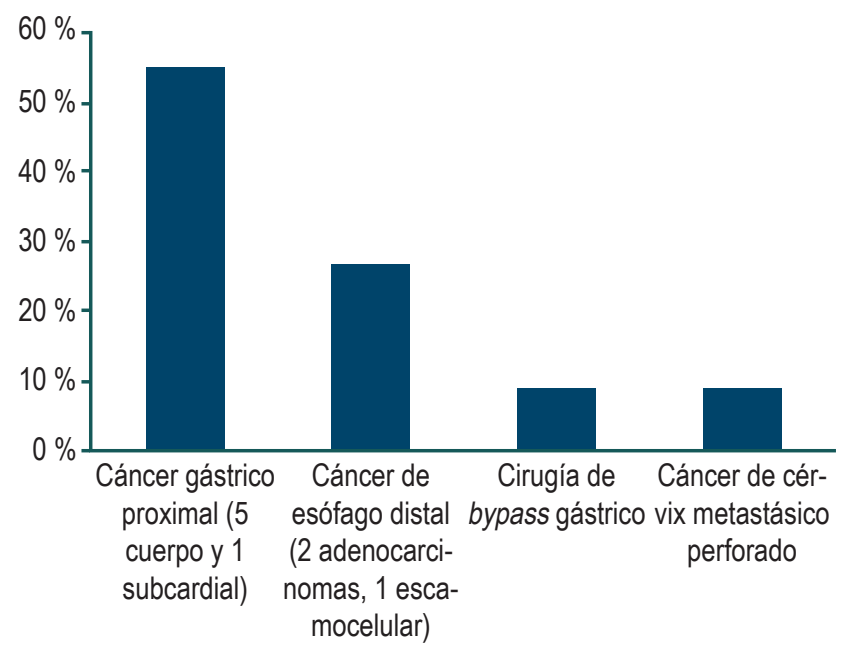

B

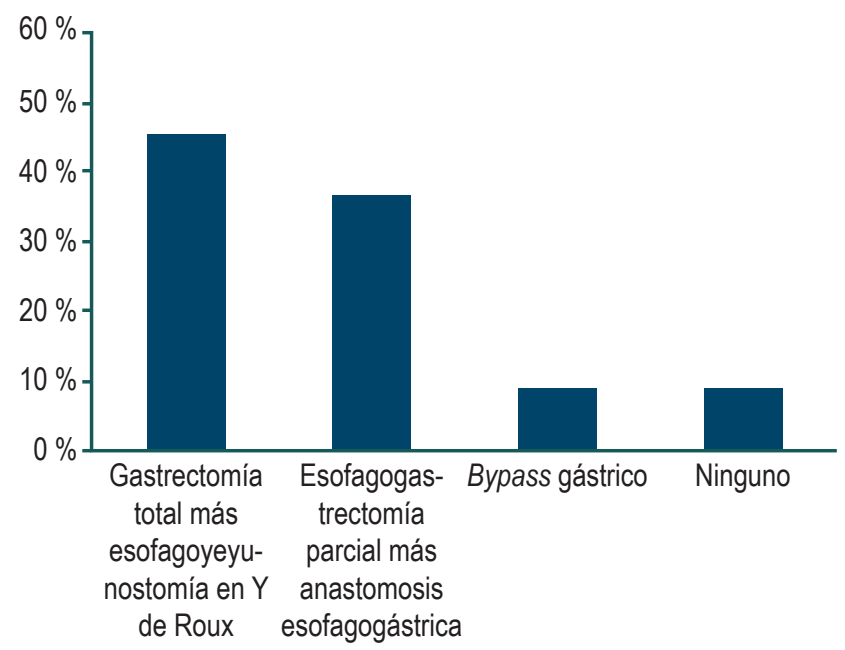

Figura 1. A. Características del diagnóstico de base. B. Manejo quirúrgico. 
Tabla 1. Características del diagnóstico clínico

\begin{tabular}{|c|c|}
\hline Hallazgo en el seguimiento & $\mathrm{n}(\%)$ \\
\hline Empiema pleural & $1(9,1)$ \\
\hline Bronquitis & $1(9,1)$ \\
\hline Neumonía & $1(9,1)$ \\
\hline Derrame pleural & $4(36,4)$ \\
\hline Derrame pleural más empiema & $1(9,1)$ \\
\hline Colección subfrénica & $1(9,1)$ \\
\hline Mediastinitis más derrame pleural & $1(9,1)$ \\
\hline Peritonitis & $1(9,1)$ \\
\hline Realización del diagnóstico & $\mathrm{n}(\%)$ \\
\hline Esofagograma & $5(45,5)$ \\
\hline Esofagograma y EVDA & $1(9,1)$ \\
\hline Esofagograma y TAC de tórax & $1(9,1)$ \\
\hline EVDA y TAC de tórax & $2(18,2)$ \\
\hline EVDA y azul de metileno & $1(9,1)$ \\
\hline Laparotomía más EVDA & $1(9,1)$ \\
\hline Tipo de fístula & $n(\%)$ \\
\hline Esofagopleural & $4(36,4)$ \\
\hline Traqueoesofágica & $1(9,1)$ \\
\hline Esofagobronquial & $1(9,1)$ \\
\hline Anastomótica & $5(45,5)$ \\
\hline Hemoglobina (g/dL) & Resultado $(n=32)$ \\
\hline Media & 10,07 \\
\hline DE & 1,47 \\
\hline Rango & $7,60-12,41$ \\
\hline Mediana & 9,9 \\
\hline
\end{tabular}

diagnóstico de fístula en la mayoría de los casos (45,5\%) con esofagograma, en 2 casos con endoscopia de vías digestivas altas (EVDA) y tomografía axial computarizada (TAC) de tórax (18,2\%), y en los 4 casos restantes con esofagograma y EVDA, esofagograma y TAC Tórax, EVDA y azul de metileno, cada uno respectivamente (Tabla 1). El tipo de fístula más reportado es el relacionado con la anastomosis esofagoentérica (45,5\%), seguido de la esofagopleural $(36,4 \%)$, para lo cual dichos pacientes fueron manejados con SMA.

En relación con el manejo endoscópico de la fístula, se encontró que el tiempo promedio de inserción del SMA desde que se realizó el diagnóstico fue de 6,5 días, y el SMA totalmente recubierto fue el más utilizado (en un 54,5\% de los casos), seguido por SMA parcialmente recubierto (en un 36,4\% de los casos) y por SMA parcialmente recubierto más OVESCO (en el 9,1 \% de los casos). Se utilizó el sistema de clip OVESCO para el cierre del defecto y se protegió este con un SMA.

Todos los procedimientos tuvieron éxito técnico y la resolución de síntomas se evidenció en el 72,7 \% de los casos. La duración del SMA in situ tuvo en promedio 33,67 días, aunque cabe resalta que este dato no se definió en 5 pacientes dado que fallecieron con el SMA in situ. La única complicación reportada fue el desplazamiento del SMA $(27,3 \%)$ y, de estos, un paciente requirió la reposición del SMA en 3 oportunidades debido a que hubo desplazamientos (Tabla 2). El promedio de la estancia hospitalaria fue 41,5 días. La resolución de la fístula se evidenció en el $63,5 \%$ de los casos. No se reportaron muertes relacionadas con la fístula, con fugas o con el implante del SMA.

De acuerdo con las características de los pacientes en los que no hubo resolución de los síntomas después del manejo endoscópico de la fístula (Tabla 3), se encontró que los 3 pacientes que no tuvieron éxito clínico fueron hombres y no tenían comorbilidades. Estos 3 pacientes no presentaron síntomas agudos, 2 tenían como diagnóstico inicial adenocarcinoma del tercio distal del esófago y el otro paciente tenía adenocarcinoma difuso del cuerpo gástrico.

En 2 casos se realizó gastrectomía total más esofagoyeyunostomía en Y de Roux, y en el otro caso se realizó esofagogastrectomía subtotal más anastomosis esofagogástrica; en 2 casos se presentó fístula esofagopleural y en el otro, fístula traqueoesofágica. Dos de los SMA utilizados en el fracaso clínico eran totalmente recubiertos y el otro, parcialmente recubierto. Además, dos de los casos presentaron desplazamiento como complicación.

Con respecto al tiempo de inserción del SMA desde el diagnóstico de la fístula, en promedio fue dos días menos en los casos de éxito que en los de fallos, y la media en el valor de hemoglobina fue menor en los pacientes que no tuvieron éxito clínico.

\section{DISCUSIÓN}

Las fístulas esofágicas tienen un amplio espectro de presentación que varía desde síntomas inespecíficos como 

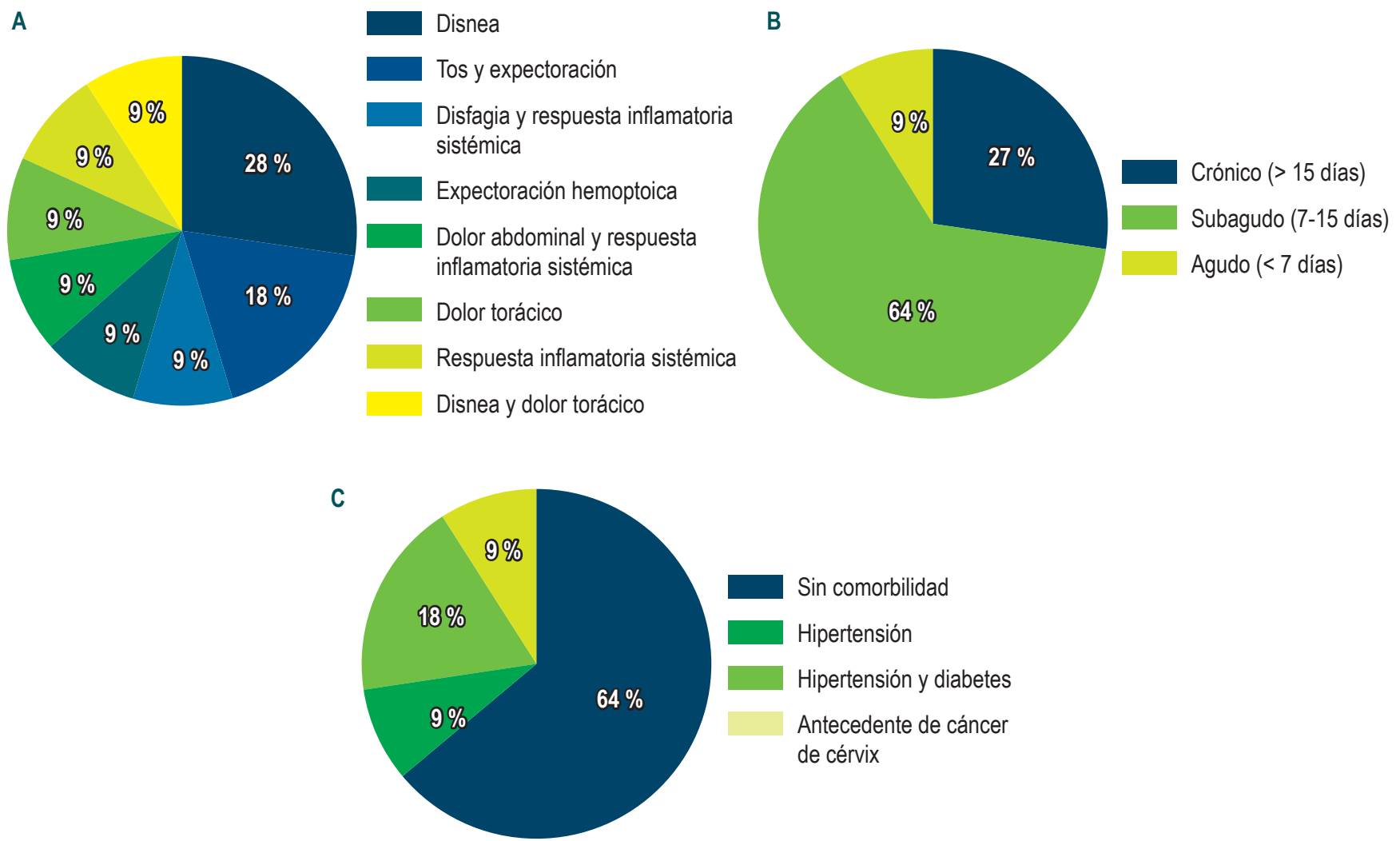

Figura 2. Características clínicas de los sujetos de estudio. A. Síntomas por los cuales consulta. B. Tiempo de evolución de los síntomas. C. Comorbilidades.

disnea o síndrome de respuesta inflamatoria sistémica (SRIS), hasta compromiso grave del paciente, debido a la sepsis secundaria a empiema o mediastinitis, por lo cual el tratamiento debe ser individualizado. Teniendo en cuenta los resultados desfavorables después de la reintervención quirúrgica en el caso de las fístulas anastomóticas, se ha desplazado cada vez más dicho manejo y se han considerado los manejos menos invasivos como las intervenciones endoscópicas con implante de clips, OVESCO o SMA, como la primera elección ${ }^{(4,5,9)}$.

Hasta el momento, no se ha realizado un consenso para un tratamiento adecuado de las fístulas esofágicas; sin embargo, se han publicado informes de casos y series pequeñas en los cuales el manejo endoscópico con SMA ha sido el pilar del tratamiento en diferentes patologías asociadas con fístula ${ }^{(14-17)}$. En nuestra serie de casos se describen situaciones de complejidad elevada como las fístulas esofagotraqueales y esofagobronquiales, que fueron manejadas por endoscopistas experimentados, lo cual explica la alta tasa de éxito técnico y la tasa de mortalidad fue baja en relación con el implante del SMA, en comparación con otras series publicadas (Tabla 2).

En esta serie de casos la tasa de mortalidad en relación con el SMA fue $0 \%$, importante en comparación con otros estudios, lo cual se puede relacionar con la no reintervención quirúrgica, que puede incrementar la mortalidad posterior al implante de un SMA debido a la condición clínica del paciente ${ }^{(14,18-20)}$. Además, otra de las justificaciones de la baja mortalidad y el éxito técnico y clínico evidenciado en nuestros pacientes se relaciona con el tiempo de implante de SMA desde el diagnóstico, que juega un papel fundamental, como se evidenció con éxito en los pacientes que fueron manejados con implante de SMA en los 2 primeros días de haberse realizado el diagnóstico, en comparación con los que recibieron dicho manejo después de 2 días, por lo que queda plenamente justificado que el implante de un SMA tempranamente reduce de forma importante la morbimortalidad de esta patología potencialmente letal. Por esta razón, una vez 
Tabla 2. Características del procedimiento quirúrgico

\begin{tabular}{|c|c|}
\hline Características del procedimiento & $\begin{array}{c}\text { Resultado } \\
(n=11)\end{array}$ \\
\hline \multicolumn{2}{|l|}{ Inserción de stent desde el diagnóstico (días) } \\
\hline - Media & 6,55 \\
\hline$-\mathrm{DE}$ & 3,47 \\
\hline - Rango & $2-12$ \\
\hline - Mediana & 7,00 \\
\hline Tipo de stent & n (\%) \\
\hline Totalmente recubierto & $6(54,5)$ \\
\hline Parcialmente recubierto & $4(36,4)$ \\
\hline Parcialmente recubierto más OVESCO & $1(9,1)$ \\
\hline Éxito técnico & $\mathrm{n}(\%)$ \\
\hline Satisfactorio & $11(100)$ \\
\hline Éxito clínico & $\mathrm{n}(\%)$ \\
\hline Resolución de síntomas & $8(72,7)$ \\
\hline Sin resolución de síntomas & $3(27,3)$ \\
\hline Duración stent in situ (días) & $\begin{array}{c}\text { Resultado } \\
(n=6)\end{array}$ \\
\hline Media & 33,67 \\
\hline DE & 45,81 \\
\hline Rango & $11-140$ \\
\hline Mediana & 62 \\
\hline Cambios del stent $\left(\mathrm{n}^{\circ}{ }^{\circ}\right)$ & $\begin{array}{c}\text { Resultado } \\
(n=11)\end{array}$ \\
\hline Media & 0,27 \\
\hline $\mathrm{DE}$ & 0,90 \\
\hline Rango & $0-3$ \\
\hline Mediana & 0,00 \\
\hline Complicaciones & $\mathrm{n}(\%)$ \\
\hline Ninguna & $8(72,7)$ \\
\hline Desplazamiento & $3(27,3)$ \\
\hline Duración de estancia hospitalaria (dias) & $\begin{array}{c}\text { Resultado } \\
(n=29)\end{array}$ \\
\hline Media & 41,55 \\
\hline DE & 59,44 \\
\hline Rango & $8-210$ \\
\hline Mediana & 21 \\
\hline Resolución de la fístula & $\mathrm{n}(\%)$ \\
\hline Resolvió & $7(63,6)$ \\
\hline Persistió & $3(27,3)$ \\
\hline Reapareció & $1(9,1)$ \\
\hline Mortalidad relacionada con la fístula, fuga o stent & $\mathrm{n}(\%)$ \\
\hline No & $11(100)$ \\
\hline Mortalidad relacionada con la patología de base & $n(\%)$ \\
\hline Sí & $8(72,7)$ \\
\hline No & $3(27,3)$ \\
\hline
\end{tabular}

que se evidencie una evolución inadecuada en un paciente, se debe evaluar lo más pronto posible la realización de una endoscopia digestiva alta o un estudio radiológico para realizar un diagnóstico y un manejo oportunos.

Se debe tener en cuenta que el manejo endoscópico de las fístulas esofágicas es complejo desde el punto de vista técnico y con amplias implicaciones en lo económico, dado que el implante del SMA no es el único procedimiento que se realiza, teniéndose en cuenta que se requieren estudios adicionales, como las EVDA repetitivas y estudios radiológicos, hasta que se obtenga un diagnóstico preciso y se tenga claro el diagnóstico y la resolución de la fístula.

Es importante tener en cuenta el tipo de SMA a utilizar. Los SMA parcial o totalmente recubiertos se utilizan en el manejo del cáncer esofágico, fístulas anastomóticas y perforaciones esofágicas iatrogénicas, con un éxito adecuado $^{(21,22)}$, pero se han planteado dudas sobre la eficacia a largo plazo de los SMA debido a sus complicaciones ${ }^{(23)}$, como el incrustamiento a la pared esofágica, por lo que el retiro endoscópico puede ser complejo ${ }^{(23-25)}$. En nuestra serie de casos en 6 pacientes se retiró el SMA sin complicaciones después de la confirmación de resolución de la fístula, teniéndose en cuenta que la extracción del SMA debe ser cuidadosa por el riesgo de perforación y broncoaspiración durante el procedimiento.

En nuestra serie de casos, el cierre de la fístula se presentó en el 63,3\%, ligeramente por debajo de lo que se describe en la literatura, lo cual se puede atribuir a la migración distal (que se presentó en el 27,3\% de nuestros casos) y al tiempo de implante del SMA después de la realización del diagnóstico de fístula; sin embargo, la mortalidad fue del $0 \%$ en relación con el implante del SMA, con una media de estancia hospitalaria de 41,5 días, lo cual se relaciona con la condición clínica de cada paciente.

Vale la pena tener en cuenta técnicas endoscópicas que se pueden utilizar de forma combinada, como el uso de cianoacrilato, sutura endoscópica, técnicas de vacío (Endo-SPONGE ${ }^{\circledast}$ ) y OVESCO en este tipo de fístulas; este último se usó en nuestro caso para el cierre del defecto y, posteriormente, se protegió con un SMA parcialmente cubierto. Las técnicas combinadas cada vez tienen mayor aceptación con tasas de éxito técnico y éxito clínico favorables, como lo describieron Thiruvengadam y colaboradores en su cohorte retrospectiva ${ }^{(26)}$.

En conclusión, con base en los resultados y lo reportado en la literatura, se puede afirmar que el manejo endoscópico de las fístulas o fugas esofágicas con SMA es una alternativa efectiva y segura, con mejoría de los síntomas, un alto cierre de las mismas y bajo riesgo de complicaciones. 
Tabla 3. Características según el éxito clínico del procedimiento quirúrgico

\begin{tabular}{|c|c|c|c|c|c|}
\hline \multirow[t]{3}{*}{ Variable } & & \multicolumn{4}{|c|}{ Éxito clínico } \\
\hline & & \multicolumn{2}{|l|}{ Sí } & \multicolumn{2}{|l|}{ No } \\
\hline & & Recuento & $\%$ & Recuento & $\%$ \\
\hline \multirow[t]{2}{*}{ Sexo } & - Hombre & 2 & 25,0 & 3 & 100 \\
\hline & - Mujer & 6 & 75,0 & 0 & 0,0 \\
\hline \multirow[t]{4}{*}{ Comorbilidad } & - Sin comorbilidad & 4 & 50,0 & 3 & 100 \\
\hline & - Hipertensión & 1 & 12,5 & 0 & 0,0 \\
\hline & - Hipertensión y diabetes & 2 & 25,0 & 0 & 0,0 \\
\hline & - Antecedente de cáncer de cérvix & 1 & 12,5 & 0 & 0,0 \\
\hline \multirow[t]{2}{*}{ Procedencia } & - Bogotá & 7 & 87,5 & 3 & 100 \\
\hline & - Amazonas & 1 & 12,5 & 0 & 0,0 \\
\hline \multirow{3}{*}{$\begin{array}{l}\text { Tiempo evolución de } \\
\text { síntomas }\end{array}$} & - Crónico & 2 & 25,0 & 1 & 33,3 \\
\hline & - Subagudo & 5 & 62,5 & 2 & 66,7 \\
\hline & - Agudo & 1 & 12,5 & 0 & 0,0 \\
\hline \multirow{9}{*}{$\begin{array}{l}\text { Diagnóstico de patología } \\
\text { inicial }\end{array}$} & - Adenocarcinoma del tercio distal del esófago & 0 & 0,0 & 2 & 66,7 \\
\hline & - Adenocarcinoma difuso de cuerpo & 1 & 12,5 & 1 & 33,3 \\
\hline & - Adenocarcinoma de tipo intestinal moderadamente diferenciado de cuerpo & 1 & 12,5 & 0 & 0,0 \\
\hline & - Adenocarcinoma de tipo intestinal mal diferenciado de cuerpo & 1 & 12,5 & 0 & 0,0 \\
\hline & - Adenocarcinoma de tipo intestinal moderadamente diferenciado subcardial & 1 & 12,5 & 0 & 0,0 \\
\hline & - Cáncer escamocelular en el tercio distal del esófago & 1 & 12,5 & 0 & 0,0 \\
\hline & - Cáncer de cérvix & 1 & 12,5 & 0 & 0,0 \\
\hline & - Obesidad & 1 & 12,5 & 0 & 0,0 \\
\hline & - Adenocarcinoma gástrico de tipo intestinal bien diferenciado de cuerpo & 1 & 12,5 & 0 & 0,0 \\
\hline \multirow[t]{4}{*}{ Manejo quirúrgico } & - Gastrectomía total más esofagoyeyunostomía en Y de Roux & 2 & 25,0 & 2 & 66,7 \\
\hline & - Esofagogastrectomía más esofagogastrostomía & 4 & 50,0 & 1 & 33,3 \\
\hline & - Bypass gástrico & 1 & 12,5 & 0 & 0,0 \\
\hline & - Ninguno & 1 & 12,5 & 0 & 0,0 \\
\hline \multirow[t]{3}{*}{ Tipo de stent } & - Totalmente recubierto & 4 & 50,0 & 2 & 66,7 \\
\hline & - Parcialmente recubierto & 3 & 37,5 & 1 & 33,3 \\
\hline & - Parcialmente recubierto más OVESCO & 1 & 12,5 & 0 & 0,0 \\
\hline \multirow[t]{4}{*}{ Tipo de fístula } & - Esofagopleural & 2 & 25,0 & 2 & 66,7 \\
\hline & - Traqueoesofágica & 0 & 0,0 & 1 & 33,3 \\
\hline & - Esofagobronquial & 1 & 12,5 & 0 & 0,0 \\
\hline & - Esofagoénterica & 5 & 62,5 & 0 & 0,0 \\
\hline \multirow[t]{2}{*}{ Complicaciones } & - Ninguna & 7 & 87,5 & 1 & 33,3 \\
\hline & - Desplazamiento & 1 & 12,5 & 2 & 66,7 \\
\hline \multirow[t]{2}{*}{ Edad (años) } & - Mediana & \multirow{2}{*}{\multicolumn{2}{|c|}{$\begin{array}{c}64,5 \\
61,25\end{array}$}} & \multirow{2}{*}{\multicolumn{2}{|c|}{$\begin{array}{l}49,00 \\
50,67\end{array}$}} \\
\hline & - Media & & & & \\
\hline \multirow{2}{*}{$\begin{array}{l}\text { Tiempo de estancia } \\
\text { hospitalaria (días) }\end{array}$} & - Mediana & \multirow{2}{*}{\multicolumn{2}{|c|}{$\begin{array}{c}23,5 \\
44,88\end{array}$}} & \multirow{2}{*}{\multicolumn{2}{|c|}{$\begin{array}{l}13,00 \\
32,67\end{array}$}} \\
\hline & - Media & & & & \\
\hline \multirow{2}{*}{$\begin{array}{l}\text { Inserción del stent desde el } \\
\text { diagnóstico (días) }\end{array}$} & - Mediana & \multirow{2}{*}{\multicolumn{2}{|c|}{$\begin{array}{l}6,0 \\
6,0\end{array}$}} & \multirow{2}{*}{\multicolumn{2}{|c|}{$\begin{array}{l}7,0 \\
8,0\end{array}$}} \\
\hline & - Media & & & & \\
\hline \multirow[t]{2}{*}{ Hemoglobina } & - Mediana & \multirow{2}{*}{\multicolumn{2}{|c|}{$\begin{array}{l}10,40 \\
10,21\end{array}$}} & 9,90 & \\
\hline & - Media & & & 9,71 & \\
\hline
\end{tabular}


1. Bemelman WA, Baron TH. Endoscopic Management of Transmural Defects, Including Leaks, Perforations, and Fistulae. Gastroenterology. 2018;154(7):1938-1946.e1. https://doi.org/10.1053/j.gastro.2018.01.067

2. Cereatti F, Grassia R, Drago A, Conti CB, Donatelli G. Endoscopic management of gastrointestinal leaks and fistulae: What option do we have? World J Gastroenterol. 2020;26(29):4198-4217. https://doi.org/10.3748/wjg.v26.i29.4198

3. Falconi M, Pederzoli P. The relevance of gastrointestinal fistulae in clinical practice: a review. Gut. 2001 Dec;49 Suppl 4(Suppl 4):iv2-10. https://doi.org/10.1136/gut.49.suppl_4.iv2

4. González-Pinto I, González EM. Optimising the treatment of upper gastrointestinal fistulae. Gut. 2001;49 Suppl 4(Suppl 4):iv22-31. https://doi.org/10.1136/gut.49.suppl_4.iv21

5. Ge PS, Thompson CC. The Use of the Overstitch to Close Perforations and Fistulas. Gastrointest Endosc Clin N Am. 2020;30(1):147-161. https://doi.org/10.1016/j.giec.2019.08.010

6. Datta V, Windsor AC. Surgical management of enterocutaneous fistula. Br J Hosp Med (Lond). 2007;68(1):28-31. https://doi.org/10.12968/hmed.2007.68.1.22652

7. Kwon SH, Oh JH, Kim HJ, Park SJ, Park HC. Interventional management of gastrointestinal fistulas. Korean J Radiol. 2008;9(6):541-9. https://doi.org/10.3348/kjr.2008.9.6.541

8. Schecter WP. Management of enterocutaneous fistulas. Surg Clin North Am. 2011;91(3):481-91. https://doi.org/10.1016/j.suc.2011.02.004

9. Dasari BV, Neely D, Kennedy A, Spence G, Rice P, Mackle E, Epanomeritakis E. The role of esophageal stents in the management of esophageal anastomotic leaks and benign esophageal perforations. Ann Surg. 2014;259(5):852-60. https://doi.org/10.1097/SLA.0000000000000564

10. Rodrigues-Pinto E, Repici A, Donatelli G, Macedo G, Devière J, van Hooft JE, Campos JM, Galvao Neto M, Silva M, Eisendrath P, Kumbhari V, Khashab MA. International multicenter expert survey on endoscopic treatment of upper gastrointestinal anastomotic leaks. Endosc Int Open. 2019;7(12):E1671-E1682. https://doi.org/10.1055/a-1005-6632

11. Ross WA, Alkassab F, Lynch PM, Ayers GD, Ajani J, Lee $\mathrm{JH}$, Bismar M. Evolving role of self-expanding metal stents in the treatment of malignant dysphagia and fistulas. Gastrointest Endosc. 2007;65(1):70-6. https://doi.org/10.1016/j.gie.2006.04.040

12. Blackmon SH, Santora R, Schwarz P, Barroso A, Dunkin BJ. Utility of removable esophageal covered self-expanding metal stents for leak and fistula management. Ann Thorac Surg. 2010;89(3):931-6; discussion 936-7. https://doi.org/10.1016/j.athoracsur.2009.10.061
13. Tuebergen D, Rijcken E, Mennigen R, Hopkins AM, Senninger N, Bruewer M. Treatment of thoracic esophageal anastomotic leaks and esophageal perforations with endoluminal stents: efficacy and current limitations. J Gastrointest Surg. 2008;12(7):1168-76. https://doi.org/10.1007/s11605-008-0500-4

14. Doniec JM, Schniewind B, Kahlke V, Kremer B, Grimm $\mathrm{H}$. Therapy of anastomotic leaks by means of covered self-expanding metallic stents after esophagogastrectomy. Endoscopy. 2003;35(8):652-8. https://doi.org/10.1055/s-2003-41509

15. Roy-Choudhury SH, Nicholson AA, Wedgwood KR, Mannion RA, Sedman PC, Royston CM, Breen DJ. Symptomatic malignant gastroesophageal anastomotic leak: management with covered metallic esophageal stents. AJR Am J Roentgenol. 2001;176(1):161-5. https://doi.org/10.2214/ajr.176.1.1760161

16. Siersema PD, Homs MY, Haringsma J, Tilanus HW, Kuipers EJ. Use of large-diameter metallic stents to seal traumatic nonmalignant perforations of the esophagus. Gastrointest Endosc. 2003;58(3):356-61. https://doi.org/10.1067/s0016-5107(03)00008-7

17. Kauer WK, Stein HJ, Dittler HJ, Siewert JR. Stent implantation as a treatment option in patients with thoracic anastomotic leaks after esophagectomy. Surg Endosc. 2008;22(1):50-3. https://doi.org/10.1007/s00464-007-9504-5

18. Urschel JD. Esophagogastrostomy anastomotic leaks complicating esophagectomy: a review. Am J Surg. 1995; 169(6):634-40. https://doi.org/10.1016/s0002-9610(99)80238-4

19. Gelbmann CM, Ratiu NL, Rath HC, Rogler G, Lock G, Schölmerich J, Kullmann F. Use of self-expandable plastic stents for the treatment of esophageal perforations and symptomatic anastomotic leaks. Endoscopy. 2004;36(8):695-9. https://doi.org/10.1055/s-2004-825656

20. Johnsson E, Lundell L, Liedman B. Sealing of esophageal perforation or ruptures with expandable metallic stents: a prospective controlled study on treatment efficacy and limitations. Dis Esophagus. 2005;18(4):262-6. https://doi.org/10.1111/j.1442-2050.2005.00476.x

21. Raijman I. Endoscopic management of esophagorespiratory fistulas: expanding our options with expandable stents. Am J Gastroenterol. 1998;93(4):496-9. https://doi.org/10.1111/j.1572-0241.1998.496_b.x

22. Ramirez FC, Dennert B, Zierer ST, Sanowski RA. Esophageal self-expandable metallic stents--indications, practice, techniques, and complications: results of a national survey. Gastrointest Endosc. 1997;45(5):360-4. https://doi.org/10.1016/s0016-5107(97)70144-5

23. Song HY, Park SI, Jung HY, Kim SB, Kim JH, Huh SJ, Kim TH, Kim YK, Park S, Yoon HK, Sung KB, Min YI. Benign and malignant esophageal strictures: treatment with a 
polyurethane-covered retrievable expandable metallic stent. Radiology. 1997;203(3):747-52.

https://doi.org/10.1148/radiology.203.3.9169699

24. Wadhwa RP, Kozarek RA, France RE, Brandabur JJ, Gluck M, Low DE, Traverso LW, Moonka R. Use of self-expandable metallic stents in benign GI diseases. Gastrointest Endosc. 2003;58(2):207-12.

https://doi.org/10.1067/mge.2003.343

25. Lee $\mathrm{SH}$. The role of oesophageal stenting in the nonsurgical management of oesophageal strictures. Br J Radiol.
2001;74(886):891-900.

https://doi.org/10.1259/bjr.74.886.740891

26. Thiruvengadam NR, Hamerski C, Nett A, Bhat Y, Shah J, Bernabe J, Kane S, Binmoeller K, Watson RR. Combination Endoscopic Therapy is Effective for Treatment of Nonbariatric Postoperative Gastroenteric Leaks. Techniques and Innovations in Gastrointestinal Endoscopy. 2021;23(2):122-8.

https://doi.org/10.1016/j.tige.2020.11.003 